\title{
An Extension of the Real Option Approach to the Evaluation of Health Care Technologies: The Case of Positron Emission Tomography
}

\author{
Paolo Pertile ${ }^{\S}$
}

2007

\begin{abstract}
This paper aims to incorporate option values into the economic evaluation of Positron Emission Tomography (PET). The installation of this equipment requires a substantial capital outlay, while uncertainty, especially regarding the possibility of new applications, is relevant, because the evidence available is still insufficient. Treating the number of examinations to provide as a stochastic variable, the costeffectiveness analysis is extended to include the value of flexibility both with respect to the timing of investment and to the size of the project. The threshold values of the stochastic variable that ensure the costeffectiveness of a PET scan according to this approach are obtained as a function of the value of the incremental effectiveness.
\end{abstract}

\footnotetext{
${ }^{\ddagger}$ This paper is an extract from my PhD thesis (Graduate School in the Economics and Finance of Public Administration (DEFAP); Università Cattolica, Milano, Università di Milano Bicocca, Università degli Studi di Milano). I am grateful to my supervisors Rosella Levaggi and Michele Moretto. I would like to thank the department of Medicine and Public Health of the University of Verona and in particular Emanuele Torri for fundamental advice on medical aspects. The data have been kindly provided by the Azienda Ospedaliera (local hospital trust) of Verona (Italy). The views expressed in this paper are those of the author and do not reflect those of the Azienda Ospedaliera of Verona.

$\S$ University of Verona.
} 


\section{Introduction}

Since the end of the 1970s the literature on real options has been challenging the standard approach to the economic evaluation under uncertainty based on net expected values. The idea is that the naïve approach fails to take into account the implicitly dynamic structure of decisions related to irreversible investments when some form of flexibility is embedded in the project. It is often the case, for instance, that the investment decision can be postponed. Building on the analogy with financial options, since the information available at different points in time is not the same, having an opportunity but not an obligation to invest has a value per se. This should be explicitly included in a comprehensive measure of the value of the project.

The impact of this extension on "capital budgeting" has been significant in the private sector ${ }^{1}$. Although the concepts of uncertainty and irreversibility are relevant independently of the perspective adopted in the evaluation, only recently have real options come to be considered in the context of costbenefit analysis $^{2}$. The potential relevance of real options for the evaluation of health care technologies has been discussed by Palmer and Smith (2000). The combination of rapid innovation and limited evidence, which is typical for most medical technologies, makes uncertainty a key issue for the economic evaluation. If there are no costs of reversal, the adoption decision can be kept separate from the decision to acquire more information (Claxton, 1999). However, irreversibility is often substantial for health care technologies. Unless the decision is actually "now or never", the static criterion based on Incremental Cost-Effectiveness Ratios (ICER) may be an inadequate tool to deal with uncertainty and irreversibility. These characteristics may coexist both at a macro-level and at the micro-level of the patient. The first case is typical for investment in new equipment, when some of the relevant variables are subject to uncertainty. An instance of irreversibility at the micro level is a treatment that, once started, induces irreversible consequences

\footnotetext{
${ }^{1}$ Dixit and Pindyck (1994) provide a comprehensive introduction to the approach. A collection of the most relevant contributions can be found in Schwartz and Trigeorgis (2001).

${ }^{2}$ See, for example, Knudsen and Scandizzo (2002).
} 
such as a progressive reduction of the effectiveness of the treatment itself or of other interventions (Lasserre, Moatti, and Soubeyran, 2006). Eckermann and Willan (2007a) show that, if a technology is immediately adopted, the costs of reversal reduce the value of additional sample information. With several jurisdictions and non rival trial-information the acquisition of new information should be optimized across jurisdictions, whereas the adoption decision should remain within jurisdictions (Eckermann and Willan, 2007b). Consistent with the real options literature, in this paper, the attention is restricted to the latter decision, and the information flow is assumed exogenous.

To the best of our knowledge no actual application of the real option approach to the evaluation of health care technologies has been published yet. The present paper aims to provide an application to the economic evaluation of Positron Emission Tomography (PET). This is a non-invasive 3-dimensional imaging technology, which requires the injection of a radiolabelled tracer. The use of radiopharmaceuticals has important consequences for the assessment of the budget and organizational impact, and hence for the determination of the cost of provision of each examination. Although several organizational modalities may be chosen for the installation, the capital cost for the equipment and the necessary infrastructures is typically high, and irreversible to a large extent. Moreover, some of the variables that are relevant for the evaluation are subject to uncertainty. For example, the number of cases for which evidence of the clinical effectiveness of PET has been proved in oncology has rapidly increased over the last few years. However, the relatively limited number of studies published so far leaves significant uncertainty as to the number of examinations that will be actually required in the future.

In the next section, the main characteristics of Positron Emission Tomography are introduced, paying particular attention to those that have an impact on costs. In Section 3 the cost-effectiveness of PET is evaluated in a dynamic setting, taking the value of flexibility into account. The focus is on the uncertainty of the number of scans required. This enables the determination of the levels of the stochastic variable that ensure cost-effectiveness in the 
form of optimal thresholds. The evaluation of the cost-effectiveness of PET for any specific type of patient is beyond the scope of this work. Therefore, the results obtained are a function of the effectiveness of the technology. Section 4 concludes the paper.

\section{Positron Emisison Tomography}

PET is a non-invasive imaging technology based on the measurement of the concentrations of positron-emitting radioisotopes within tissue. This requires injection of a radiolabelled tracer. In cancer, the most common radiolabelled tracer is FDG, which is glucose labelled with 18Fluorine. PET imaging provides information about the level of biological activity in a lesion and can detect cancers due to their high rate of glucose utilization. Unlike other forms of imaging such as magnetic resonance imaging (MRI), computed tomography (CT) or ultrasound techniques, PET provides functional rather than anatomical information. Since malignancies can cause abnormalities of blood flow or metabolism before anatomical changes are apparent, this technique enables early diagnosis of cancer and improved staging, before metastatic spread occurs. At present, over $90 \%$ of applications are in oncology, but it is also used in cardiology and neurology.

Although the basic research for the production of short-lived isotopes like those used for PET scans dates back to the 1930s, the first scanner was developed in 1974. That equipment could only enable brain scans and the complexity of procedures for the production of radioisotopes required highly experienced and qualified staff. The first commercial PET scanner for whole body scan was developed in 1990. Since 2002, the CT anatomic information may be combined with the functional image of PET in one scanner (CTPET). The development of the technology through the years has reduced the complexity of procedures and enabled the achievement of higher quality imaging in less time. Meanwhile, although the development had initially been focussed on applications in neurology and cardiology, oncology has become the main field of use.

Despite the growing diffusion of this technology and the improvement of its 
performances that has enabled the shift from research to clinical use, the capital cost for the equipment and the dedicated facilities that are needed is still high. Moreover, once the project is started, the costs that must be borne independently of how many scans are performed are also substantial. This is mainly due to the need to hire highly qualified and experienced staff. Given these characteristics of the investment, the number of scans provided is a major determinant of the cost of each examination and hence a key variable for the economic evaluation. In the last few years, the number of cases for which PET has showed better performances than more standard imaging technologies has been increasing. The evidence of the clinical effectiveness of PET, however, is still limited. Most experts foresee that this trend will continue for some time, but, lacking evidence, the evolution is by definition uncertain. Uncertainty on the number of scans is reflected in the cost per scan, and possibly in other variables.

The combination of a large degree of irreversibility and relevant uncertainty seems to make PET an attractive application for the extension of real options to the evaluation of health technologies. The relevance of these issues has been qualitatively discussed in several evaluation studies, but the standard approach does not provide adequate tools to deal with them ${ }^{3}$. Moreover, a further source of flexibility besides the timing of investment may be considered. This is related to the size of the project. In particular, three alternatives are available for the provision of PET scans. With a minor capital outlay, the provider may decide to rent a scanner for a number of days per week and buy the tracer (Mobile PET). Alternatively, with an intermediate capital cost, the provider may own the scanner but still buy the tracer from another site. The maximum distance from the location where the radiopharmaceutical is produced is limited due to its relatively short half-life ${ }^{4}$. Finally, with the highest capital outlay, it is possible to produce the tracer on

\footnotetext{
3 "HTAs from around the world agree that there is insufficient evidence to fully recommend PET imaging as a standard technique in cancer management at this time. However, improvements in diagnostic accuracy compared with other imaging modalities and weak evidence of change in patient management from case series, indicate that PET is' potentially' clinically and cost effective" (Brandbury et al., 2002, 10-7).

${ }^{4}$ The half-life of the most common tracer (FDG) is about 110 minutes.
} 
the same site where the scan is provided. The additional price to pay in this case is not only that of extra equipment (cyclotron) but also the sunk cost of appropriate infrastructures needed for the production. The advantages lie in the organizational autonomy, which also permits a larger number of scans per unit of time, and a lower variable cost per scan. The investment can be expanded sequentially, as more information becomes available. In the following section the attention will be restricted to the second and third alternatives, because Mobile PET has been chosen in a very limited number of cases and therefore obtaining the relevant data is complicated. We will refer to the alternatives under consideration respectively as PET scanner and Fully Equipped PET.

\section{Cost-effectiveness of PET}

In this section, the societal perspective is adopted in the economic evaluation of PET. The objective is to incorporate the value of flexibility into costeffectiveness analysis. Several economic evaluations of the use of PET for the diagnosis and staging of specific diseases have been published ${ }^{5}$. The objective of the present paper is not to obtain an ICER for any specific use of PET, but rather to set up a model that enables the taking of real options into account in the evaluation. Therefore, the emphasis will be more on methods than on results. This may also be seen as an attempt to replace the standard approach to evaluation, which is intrinsically static, with a dynamic one.

In order to determine the optimal timing of investment in this new setting, we need to shift from a cost-effectiveness ratio to some measure of value of the project. The value per unit of time (year) function has then the Net Incremental Benefit (NIB) shape:

$$
\begin{gathered}
R_{p t}=\left(\lambda e-c_{p}\right) n_{p}(t)-f_{p} \\
R_{f t}=\left(\lambda e-c_{f}\right) n_{f}(t)-f_{f}
\end{gathered}
$$

\footnotetext{
${ }^{5}$ See Müller et al. (2002) for an overview.
} 
The first subscript refers to the type of project, the alternative being between a simple PET scanner (hereinafter P) and a Fully Equipped Pet (FEP). $\lambda$ is the standard cut-off level for the ICER, which should be interpreted as a measure of the value of effectiveness in this context. The incremental effectiveness and cost per scan are respectively $e$ and $c$, whereas $f_{p}$ and $f_{f}$ denote fixed costs per year for each alternative. The number of scans per year is denoted by $n(t)$ and it is assumed to be stochastic. In order to obtain an analytic solution to the model, we assume that $R_{p t}$ and $R_{f t}$ are linear functions of the stochastic variable. This requires us to isolate the fixed cost component for $\mathrm{P}$ and FEP. Therefore $c_{p}$ and $c_{f}$ in this case may not be interpreted as the usual numerator of the corresponding ICER, because they do not include capital and the other fixed costs of $\mathrm{P}$ and FEP.

A genuine measure of effectiveness may only be obtained by setting-up appropriate models for each application considered, which enable the shift from the sensitivity/specificity of the imaging to the impact on some measure of health outcome. On the cost side, moreover, differences among applications may arise in connection with different lengths of scans. In some cases, the technology to use for an appropriate comparison may also differ. Differences in costs, however, seem negligible to a large extent, and therefore they will be ignored in what follows. Since the objective of this paper is not to provide a detailed analysis of the cost-effectiveness of PET for any specific type of patient, but rather to suggest how the economic evaluation might be extended to include option values, the specific estimate of effectiveness will be ignored and it will only appear as a parameter. Hence, the results will also be a function of $e$. This, however, does not prevent us from pursuing our objective.

Incremental costs and effectiveness are estimated with respect to CT, as is most often done in the literature. The assumption we make is that the number of scans provided in year $t$ is the only relevant variable subject to uncertainty. Given the size of fixed and capital costs, this also looks like the most crucial variable by far.

The stochastic variable is assumed to follow a geometric Brownian motion 
with positive drift:

$$
d n(t)=\mu n(t) d t+\sigma n(t) d w(t)
$$

The first term on the right hand side of eq. (3) is a deterministic component that implies a constant increment $(\mu)$ per period in percentage terms. The second term is the purely stochastic part, embedded in the standard Wiener process $w(t)$. This is a Markov process such that $E\left[d w_{t}\right]=0$, and $E\left[d w_{t}^{2}\right]=d t$. This implies that over a finite time interval $t$, the change in the logarithm of $n$ is normally distributed with mean $\left(\mu-\frac{1}{2} \sigma^{2}\right) t$ and variance $\sigma^{2} t$. This is consistent with the intuition that uncertainty is likely to be greater, the longer the time to prediction.

The possibility that $n$ falls to zero and stays there forever is also taken into account. This could be a consequence of an innovation that makes PET obsolete. This event is assumed to follow a Poisson process with parameter $\delta$.

This kind of stochastic process enables us to solve the model analytically. In the mean time, a geometric Brownian motion seems to approximate fairly well the uncertainty of the number of patients to treat for a number of reasons. First, the positive trend component may reflect the widespread belief that the recently observed tendency towards an increase in the proportion of cancers for which PET is appropriate will continue in the future as more evidence becomes available. A constant increase, in percentage rather than in absolute terms, seems to fit the expectations better. This is connected to the idea that besides clinical effectiveness, the referral behaviour of physicians also plays a role, and this is likely to increase after the adoption of a new technology in an area. Moreover, follow-up examinations also have to be taken into account. Of course, there is a limit to the number of examinations that may be performed with one scanner in period $t$. This limit is different for $\mathrm{P}$ and FEP and it will be respectively indicated by $\bar{n}_{p}$ and $\bar{n}_{f}$. In particular, $\bar{n}_{f}$ is greater than $\bar{n}_{p}$, as a result of the time needed for transportation when the tracer is produced on a different site $^{6}$. The assumption

\footnotetext{
${ }^{6}$ This is the reason why $n_{p}$ and $n_{f}$ appear as different variables in eqs. (1) and (2)
} 
we make is that $n(t)$ follows the geometric Brownian process up to these levels and remains constant afterwards. These limits contribute somewhat to make the process in eq. (3) a better approximation of reality, although the price for this is some additional complication in the solution.

\subsection{Optimal Stopping}

The type of problem that will be solved is referred to as "optimal stopping" in the real option literature (Dixit and Pindyck, 1994). The idea is that at any point in time the value of immediate investment (stopping) is compared with the expected value of waiting $d t$ (continuation), given the information available at that point in time (the value of the stochastic variable) and the knowledge of the process. The value of projects $\mathrm{P}$ and FEP may be written as,

$$
\begin{aligned}
& V_{p}(n, t)=\max \left[\Omega_{p}(n, t), \frac{1}{1+\rho d t} E\left[V_{p}(n+d n, t+d t) \mid n\right]\right] \\
& V_{f}(n, t)=\max \left[\Omega_{f}(n, t), R_{p}(n, t)+\frac{1}{1+\rho d t} E\left[V_{f}(n+d n, t+d t) \mid n\right]\right]
\end{aligned}
$$

where, $\Omega(n, t)$ is the expected value of investing at time $t$, when the stochastic variable is equal to $n$. The social discount rate is $\rho$. The following assumption is made $^{7}$ :

$$
\rho+\delta>\mu
$$

$V_{p}$ may be interpreted as the value of the opportunity to invest in $\mathrm{P}$, which is the maximum between the expected values of "killing" the option to invest immediately, and waiting $d t$, thus keeping the option alive. $V_{f}$ is the value of the opportunity to invest in FEP, given that the scanner is already in operation. Hence, in this case, $R_{p}(n, t)$ enters the continuation value. The objective is to determine the values of the stochastic variable $n_{p}^{*}$ and $n_{f}^{*}$ that should trigger investment in $\mathrm{P}$ and FEP, or, in other words, that make a scan provided respectively with $\mathrm{P}$ and FEP cost-effective.

although the underlying stochastic process is the same.

${ }^{7}$ This assumption is standard in the real options literature. See Dixit and Pindyck (1994). 
We assume an infinite time horizon for the project ${ }^{8}$. In other words, once the project is started, there is some sort of commitment to provide PET scans as long as the technology is not displaced by some innovation, i.e. the number of scans required falls to zero. This seems consistent with the attitude of most providers, particularly in the public sector, towards the adoption of new technologies.

The problem will be solved using the dynamic programming approach. We work backwards, starting from the definition of the value function for FEP. From the technical point of view, there are four values of the stochastic variable that induce changes in the relevant form of the value function: $n_{p}^{*}$, $n_{f}^{*}, \bar{n}_{p}, \bar{n}_{f}$. These are typically referred to as barriers in the real option literature $^{9}$. The first two are endogenous barriers and the determination of those levels is the objective of the solution to the optimal stopping problem. However, $\bar{n}_{p}$ and $\bar{n}_{f}$ are exogenous and we assume they are absorbing barriers. In other words, $n$ is stochastic as long as it is smaller than the relevant upper limit $\bar{n}_{p, f}$. Once this value is reached, $n$ remains fixed at that level. The value function corresponding to the investment in FEP, therefore, takes on two different forms above and below $\bar{n}_{f}$. In the first case, the value is simply the present value of a constant payoff over the length of the project FEP:

$$
\bar{F}(n)=\frac{\bar{n}_{f}\left(\lambda e-c_{f}\right)-f_{f}}{\rho+\delta}-\Phi_{f}-I_{f}
$$

We assume that the fixed costs $f_{f}$ and $f_{p}$ must be paid only as long as the equipment is in operation $(n>0) . I_{f}$ and $\Phi_{f}$ denote respectively the capital cost of starting a FEP, and the expected future costs of investment:

$$
\Phi_{f}=\sum_{s=1}^{\infty} I_{p} e^{-10 s \delta}(1+\rho)^{-10 s}+\sum_{s=1}^{\infty}\left(I_{f}-I_{p}\right) e^{-20 s \delta}(1+\rho)^{-20 s}
$$

\footnotetext{
${ }^{8}$ This assumption is often made in the real options literature, because it simplifies the analysis. In our case, as will be explained below, the life of the project implicitly determines the life of the options in some cases. With a finite time horizon, the option values could not be determined analytically, without introducing some degree of approximation (Barone-Adesi and Whaley, 1986).

${ }^{9}$ See Dixit (1994).
} 


$$
=I_{p}\left[\frac{1}{\left(e^{\delta}(1+\rho)\right)^{10}-1}\right]+\left(I_{f}-I_{p}\right)\left[\frac{1}{\left(e^{\delta}(1+\rho)\right)^{20}-1}\right]
$$

where, $I_{p}$ is the capital cost for P. $\Phi_{f}$ enters the value function because the physical life of the installation is limited, whereas the project life is assumed infinite. In particular, the estimated life is 10 years for a scanner and 20 years for the other installations of a $\mathrm{FEP}^{10}$. Therefore, they will have to be replaced in the future, provided $n$ has not fallen to zero yet. The difference between $I_{f}$ and $\Phi_{f}$ is that the first is a sunk cost, whereas the capital costs that enter $\Phi_{f}$ will only be paid if the technology is still in use when the equipment must be replaced. For this reason, it is useful to keep these two components separate.

Below $\bar{n}_{f}$, the value function is (see the Appendix):

$$
F(n)=D_{1} n^{\beta}+\frac{\left(\lambda e-c_{f}\right) n}{\rho+\delta-\mu}-\frac{f_{f}}{\rho+\delta}-\Phi_{f}-I_{f}
$$

where, $\beta>1$ is the positive solution of the following equation:

$$
\frac{1}{2} \sigma^{2} \beta^{2}+\left(\mu-\frac{1}{2} \sigma^{2}\right) \beta-(\rho+\delta)=0
$$

The structure of eq. (6) is standard in this kind of literature. The linear part (in $n$ ) is the expected value ignoring the barrier. In this case, it is the value that would be obtained assuming that $n$ may grow without bounds (see the Appendix). $D_{1} n^{\beta}$ picks up the impact on the value of the possibility that a barrier is hit. Since in this case the barrier prevents further increases in $n$, this is a negative component. The value of the constant associated with this term is (See the Appendix):

$$
D_{1}=-\frac{\bar{n}_{f}^{1-\beta}\left(\lambda e-c_{f}\right) \mu}{(\rho+\delta)(\rho+\delta-\mu)}
$$

We can now take one step backwards to the case where there has not yet

\footnotetext{
${ }^{10}$ This is why after 10 years the scanner has to be replaced also in a FEP (eq. 5).
} 
been any investment in FEP, merely in P. Again, a distinction must be made between the two cases corresponding to $n<\bar{n}_{p}$ and $n=\bar{n}_{p}$. In the latter case, the value function is:

$$
\bar{P}(n)=K_{1} n^{\beta}+\frac{\bar{n}_{p}\left(\lambda e-c_{p}\right)-f_{p}}{\rho+\delta}-\Phi_{p}-I_{p}
$$

where,

$$
\Phi_{p}=\sum_{s=1}^{\infty} I_{p} e^{-10 s \delta}(1+\rho)^{-10 s}=I_{p}\left[\frac{1}{\left(e^{\delta}(1+\rho)\right)^{10}-1}\right]
$$

The value of the status quo (linear component) in eq. (9) is now deterministic, because without the expansion of the investment to FEP, no uncertainty is encountered on $n$. In this case, $K_{1} n^{\beta}$ is the value of the opportunity to expand the investment to FEP. The value of $K_{1}$, which will be explicitly determined below, is positive in this case.

For values of the stochastic variable smaller than $\bar{n}_{p}$, in principle, both types of barriers could play a role. The value function in this case would be (Appendix):

$$
P(n)=B_{1} n^{\beta}+C_{1} n^{\beta}+\frac{\left(\lambda e-c_{p}\right) n}{\rho+\delta-\mu}-\frac{f_{p}}{\rho+\delta}-\Phi_{p}-I_{p}
$$

The first term $\left(B_{1} n^{\beta}\right)$ may be interpreted as the value of the opportunity to expand the project to FEP, while $C_{1}$ has the same interpretation as $D_{1}$ in $F(n)$. The value of $B_{1}$ will be determined in the following sub-section, whereas it is showed in the Appendix that the value of $C_{1}$ is the following:

$$
C_{1}=-\frac{\bar{n}_{p}^{1-\beta}\left(\lambda e-c_{p}\right) \mu}{(\rho+\delta)(\rho+\delta-\mu)}
$$

In practice, whether $\bar{P}(n)$ or $P(n)$ is relevant depends on whether the shift from $\mathrm{P}$ to FEP occurs after or before $\bar{n}_{p}$ is reached. As it is showed in the next section, given our parameter values, the second case never occurs ${ }^{11}$. Bearing in mind that there will be no investment in FEP for $n<\bar{n}_{p}$, the

\footnotetext{
${ }^{11}$ This is true for any value of $\lambda$ and $e$.
} 
value function for $n_{p}^{*} \leq n<\bar{n}_{p}$ is (See the Appendix):

$$
\widetilde{P}(n)=H_{1} n^{\beta}+\frac{\left(\lambda e-c_{p}\right) n}{\rho+\delta-\mu}-\frac{f_{p}}{\rho+\delta}-\Phi_{p}-I_{p}
$$

with,

$$
H_{1}=K_{1}-\frac{\bar{n}_{P}^{1-\beta}\left(\lambda e-c_{P}\right) \mu}{(\rho+\delta)(\rho+\delta-\mu)}
$$

The exponential term is related to the possibility that $\bar{n}_{p}$ is reached. When this happens, the option to expand the investment to FEP is obtained (first term in eq. (14)), but the number of scans stops growing (second term in eq. (14)).

The last step backwards leads to the definition of the value function before any investment is made. The concepts and techniques involved are the same as above, but now the situation is of the new market rather than existing market type (Dixit and Pindyck, 1994). In other words, there is no current payoff, and therefore the value is only the opportunity to invest:

$$
W(n)=A_{1} n^{\beta}
$$

\section{$3.2 \quad$ Results}

The value functions derived in the previous sub-section are plotted in Fig. $1^{12}$.

Since the objective is optimization in a dynamic setting, each of them is relevant over the range of values where it ensures the highest value. For sufficiently low values of $n$, the highest function is $W(n)$ and therefore neither $\mathrm{P}$ nor FEP are cost-effective ${ }^{13}$. This is the case up to $n_{p}^{*}$. The figure shows how this point is determined. This is done by imposing the so called value matching and smooth pasting conditions. This means that the functions $W(n)$ (no scanner) and $\widetilde{P}(n)$ (scanner only) must be equal (value matching) and have the same derivative (smooth pasting) in $n_{p}^{*}$. This ensures that the

\footnotetext{
${ }^{12}$ The figure focuses on the functions that turn out to be relevant for our parameter values. Hence, $P(n)$ is not showed in the figure. See also Fig. 2.

${ }^{13}$ Recall that $\bar{P}(n)$ is relevant only for $n>\bar{n}_{p}$.
} 
Figure 1: Value Functions

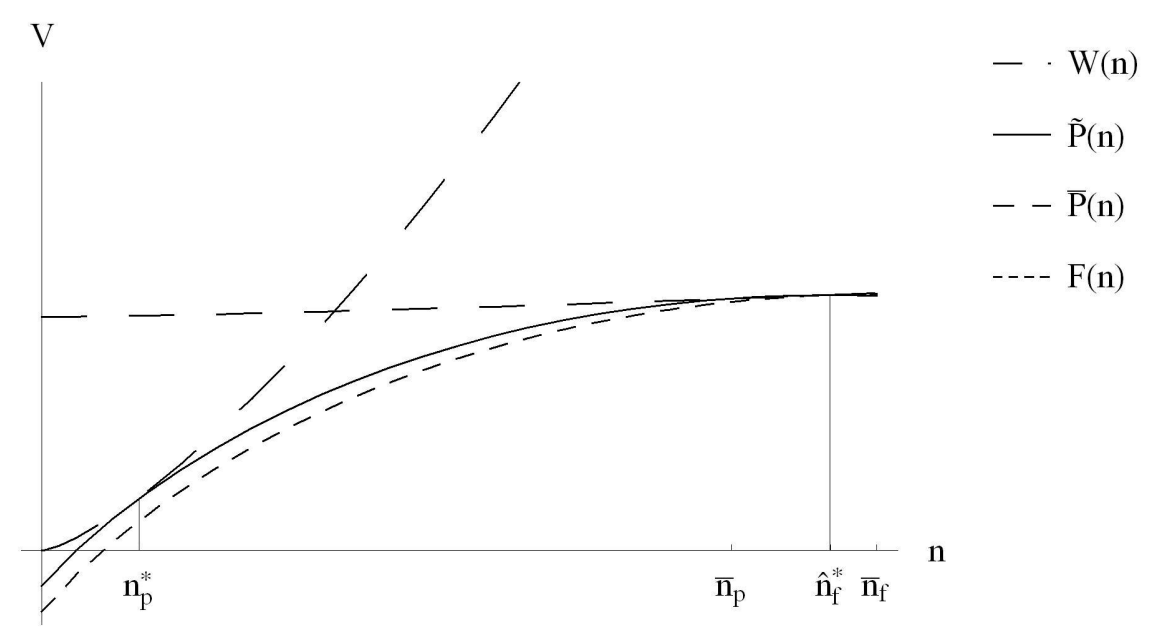

value function is continuous and differentiable up to $\bar{n}_{f}$. These restrictions make a closed form solution for $n_{p}^{*}$ and the constant $A_{1}$ possible:

$$
\begin{gathered}
n_{p}^{*}=\left(\frac{\beta}{\beta-1}\right)\left(\frac{\rho+\delta-\mu}{\lambda e-c_{p}}\right)\left[\frac{f_{p}}{\rho+\delta}+\Phi_{p}+I_{p}\right] \\
A_{1}=H_{1}+\frac{\left(n_{p}^{*}\right)^{1-\beta}}{\beta}\left[\frac{\lambda e-c_{p}}{\rho+\delta-\mu}\right]
\end{gathered}
$$

The term that typically picks up the effect of the option value on the timing of investment in eq. (16) is $\frac{\beta}{\beta-1}$. Since $\beta>1$, the effect is an increase of the so called optimal threshold. Nothing, in principle, prevents $n_{p}^{*}$ from exceeding the upper bound $\bar{n}_{p}$. If this is the case, $\mathrm{P}$ is not cost-effective for any of the feasible levels of activity.

Moving towards higher values of $n$, the same approach may be used to determine $n_{f}^{*}$. This time, however, two cases may occur. In the first situation, imposing value matching and smooth pasting between $P(n)$ and $F(n)$ a value $n_{f}^{*}<\bar{n}_{p}$ is obtained. If this is the case, the threshold for cost-effectiveness 
of FEP is:

$$
n_{f}^{*}=\left(\frac{\beta}{\beta-1}\right)\left(\frac{\rho+\delta-\mu}{c_{p}-c_{f}}\right)\left[\frac{f_{f}-f_{p}}{\rho+\delta}+\Phi_{f}-\Phi_{p}+I_{f}-I_{p}\right]
$$

with,

$$
B_{1}=D_{1}-C_{1}+\frac{\left(n_{f}^{*}\right)^{1-\beta}}{\beta}\left(\frac{c_{p}-c_{f}}{\rho+\delta-\mu}\right)
$$

Given our parameter values, this number exceeds $\bar{n}_{p}$. In other words, $P(n)$ lies always above $F(n)$ for $n<\bar{n}_{p}$ (Fig. 2).

Figure 2: Value Functions

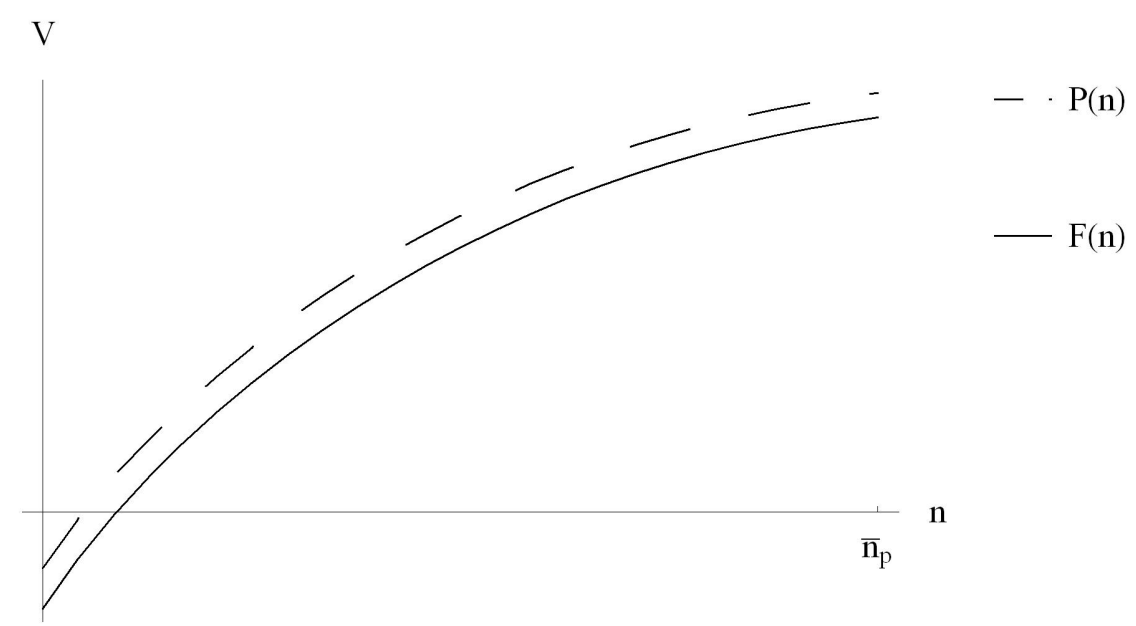

This is the reason why the relevant function in the range $n_{p}^{*} \leq n<\bar{n}_{p}$ is $\widetilde{P}(n)$. It should be observed, that the threshold $n_{f}^{*}$ is independent of $\lambda e$. This because it makes no difference in terms of effectiveness whether the scan is provided with $\mathrm{P}$ or FEP. Since the number of scans provided is also the same for $\mathrm{P}$ and FEP up to $\bar{n}_{p}$, the convenience to shift to FEP depends merely on costs.

Given that it is never optimal in our case to invest below P's capacity limit, the relevant comparison for the determination of the threshold for project FEP is between $\bar{P}(n)$ and $F(n)$. The usual restrictions yield the following 
value of the threshold and the corresponding constant for this case:

$$
\begin{gathered}
\widehat{n}_{f}^{*}=\left(\frac{\beta}{\beta-1}\right)\left(\frac{\rho+\delta-\mu}{\lambda e-c_{f}}\right)\left[\frac{\left(\lambda e-c_{p}\right) \bar{n}_{p}}{\rho+\delta}+\frac{f_{f}-f_{p}}{\rho+\delta}+\Phi_{f}-\Phi_{p}+I_{f}-I_{p}\right] \\
K_{1}=D_{1}+\frac{\left(\widehat{n}_{f}^{*}\right)^{1-\beta}}{\beta}\left(\frac{\lambda e-c_{f}}{\rho+\delta-\mu}\right)
\end{gathered}
$$

As for the threshold of project $\mathrm{P}$, it may be the case that both $n_{f}^{*}$ and $\widehat{n}_{f}^{*}$ exceed the maximum $\bar{n}_{f}$ and therefore FEP is never the optimal option. Moving backwards, this would imply that $K_{1}$ in eq. (9) (and $B_{1}$ in eq. (11)) must be set equal to zero. However, it may be ascertained that this does not affect the determination of the optimal threshold $n_{p}^{*}$. In this situation, as long as $n_{p}^{*}<\bar{n}_{p}$, it would be optimal to invest only in $\mathrm{P}$.

The overall value function for our case may be written in more compact notation as follows:

$$
V(n)= \begin{cases}A_{1} n^{\beta} & \text { for } n<n_{p}^{*} \\ H_{1} n^{\beta}+\frac{\left(\lambda e-c_{p}\right) n}{\rho+\delta-\mu}-\frac{f_{p}}{\rho+\delta}-\Phi_{p}-I_{p} & \text { for } n_{p}^{*} \leq n<\bar{n}_{p} \\ K_{1} n^{\beta}+\frac{\bar{n}_{p}\left(\lambda e-c_{p}\right)-f_{p}}{\rho+\delta}-\Phi_{p}-I_{p} & \text { for } \bar{n}_{p} \leq n<\widehat{n}_{f}^{*} \\ D_{1} n^{\beta}+\frac{\left(\lambda e-c_{f}\right)}{\rho+\delta-\mu}-\frac{f_{f}}{\rho+\delta}-\Phi_{f}-I_{f} & \text { for } \widehat{n}_{f}^{*} \leq n<\bar{n}_{f} \\ \frac{\bar{n}_{f}\left(\lambda e-c_{f}\right)-f_{f}}{\rho+\delta}-\Phi_{f}-I_{f} & \text { for } n \geq \bar{n}_{f}\end{cases}
$$

Cost estimates are based on data provided by the local hospital trust, where a project to install either a PET scanner or a FEP is under discussion. Both direct and overhead costs are included. Savings from avoided unnecessary interventions are not considered. The estimated capital costs are around 3.8 million and 8.4 million euros, respectively for $\mathrm{P}$ and FEP. Other fixed costs are also higher for the second option, being slightly above 1 million euros versus 660,000 euros.

Given that the perspective is societal, and in consideration of the assumptions made above, our hypothesis is that the variable cost when the tracer has to be bought from another site is not the full price, but the cost of 
production plus the estimated cost of transportation. Implicit is the hypothesis that the tracer may actually be bought from another FEP. If, for some reasons, the radiopharmaceutical cannot be bought from a distance compatible with transportation, investment in $\mathrm{P}$ is not an option at all. As an intermediate case, if we more strictly adopt the viewpoint of a regulator, it may be that purchases can only be made from a different authority. If the perceived cost is the price of the tracer, then $\mathrm{P}$ becomes a comparatively more expensive alternative ${ }^{14}$. The estimated cost of transportation is 80 euros. Due to the difficulty in obtaining detailed estimates for CT, the reimbursement price has been used as a proxy for the full cost of a CT scan (391 euros).

Figure 3: Optimal Thresholds

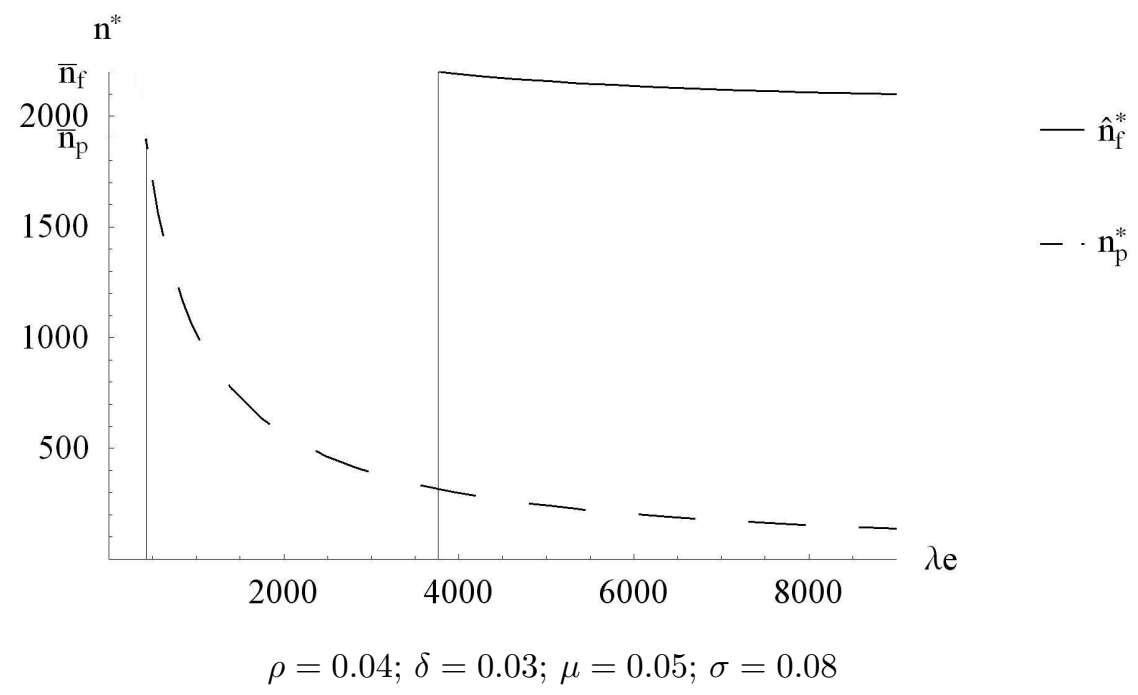

Fig. 3 shows the cost-effectiveness thresholds for P and FEP, calculated taking the value of flexibility into account, as a function of the incremental monetary value of one examination. More precisely, in the case of $\widehat{n}_{f}^{*}$, if,

\footnotetext{
${ }^{14}$ The estimated cost of a dose of the tracer bought from another FEP is around 340 euros.
} 
given the value $\lambda e$, the number of scans per year is at least as high as this threshold, option FEP is the most cost-effective.

The minimum value of a scan that ensures cost-effectiveness of the examination provided with $\mathrm{P}$ is 418 euros. In this case, the scanner should operate at full capacity. As explained above, given our data, the advantage in terms of variable costs of FEP is not large enough to compensate for the higher capital cost, as long as the number of scans provided is the same $\left(n \leq \bar{n}_{p}\right)$. When the scanner's upper bound is reached, a larger number of scans can be provided if a FEP is installed. Therefore, the optimal threshold for the cost-effectiveness of FEP in the range $\bar{n}_{p} \leq n \leq \bar{n}_{f}$ depends again on $\lambda e$. In this case, the monetary value of incremental effectiveness must be at least 3,750 euros.

As explained above, these results are obtained assuming that the additional variable cost for $\mathrm{P}$, in comparison with $\mathrm{FEP}$, is just the cost of transportation. If this assumption were relaxed and the full price of a dose of the tracer were considered, the difference between the thresholds would be reduced.

\subsection{Comparisons}

We end the analysis with a brief discussion of the quantitative impact of adopting the approach that has been discussed in this paper, instead of the standard ICER criterion, for the economic evaluation of PET. In Fig. 4, the trend component has been set equal to zero in order to highlight the size of the effect of the option value purely related to uncertainty. We restrict our attention to the comparison between the thresholds for project $\mathrm{P}^{15}$. The ratio between the two thresholds is equal to $\frac{\beta}{\beta-1}=1.24$ (see eq. (16)). In other words, taking the value of flexibility into account results in a $24 \%$ increase in the number of patients that ensure cost-effectiveness, for a given $\lambda e^{16}$.

\footnotetext{
${ }^{15}$ In the case of FEP, the comparison would not be fully homogeneous because, while in our approach the expansion to FEP takes place after the investment in $\mathrm{P}$, the typical ICER would be based on the comparison between a scan provided with FEP and CT.

${ }^{16}$ This difference is obtained assuming that the possibility that $n$ falls to zero is taken into account also in determining the standard ICER. Otherwise, our threshold would be
} 
Figure 4: Optimal Thresholds

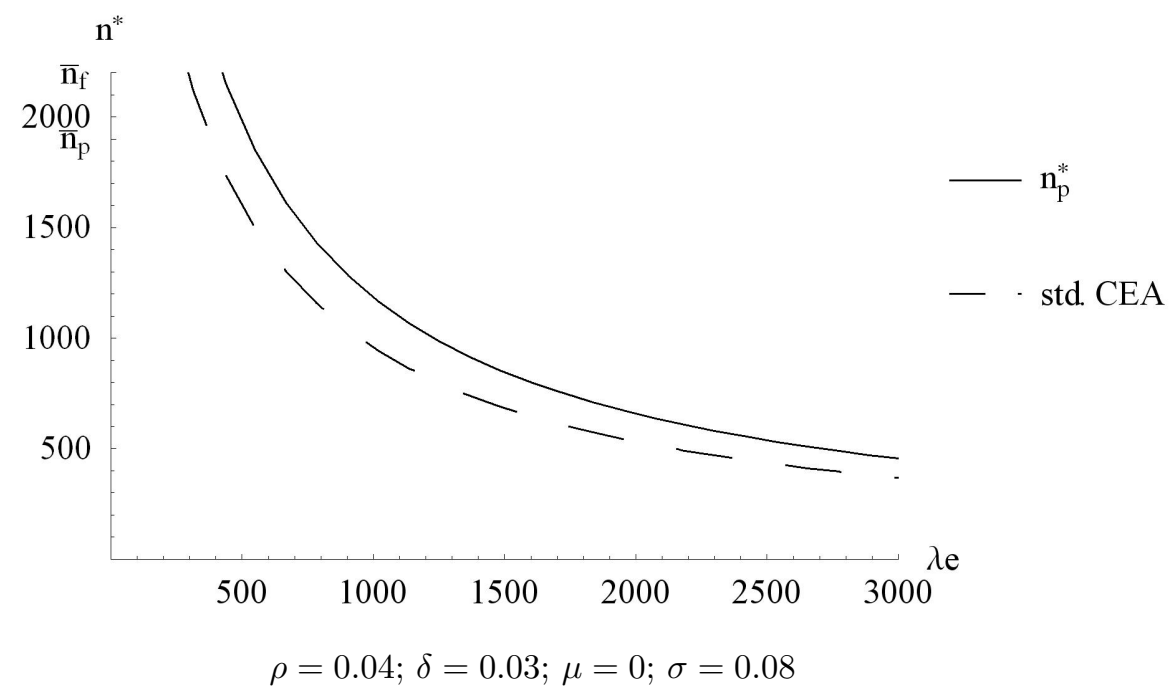

With a positive drift component, the difference between the standard ICER criterion and the one we suggest is twofold. On the one hand, there is the effect of taking the value of the opportunity to postpone the investment, given uncertainty, into account. This is best highlighted by setting $\mu=$ 0 . On the other hand, there is the effect of shifting from a completely static criterion to a dynamic approach. For example, if $\mu$ is set equal to 0.03, departing from standard ICER analysis would yield a $10 \%$ increase in the threshold. In this case, the difference is smaller, because the dynamic analysis anticipates the increase in $n$ given by the deterministic component of the geometric Brownian motion. With $\mu=0.05$, the difference is further reduced to $6 \%$.

2.2 times the ICER threshold. 


\section{Conclusion}

The aim of this paper is to incorporate option values into the economic evaluation of a specific health care technology. The relevance of this extension for irreversible investments with uncertain returns has already been demonstrated in other sectors. The typical characteristics of new medical technologies suggest that this could also be an interesting field of application. The technology that has been chosen for this application is Positron Emission Tomography (PET). This is an innovative imaging technology, mainly used in oncology, which requires the installation of expensive equipment and high fixed costs once it is in operation. Due to the scarcity of evidence available, there is substantial uncertainty over the number of types of cancer for which the appropriateness of PET will be demonstrated and hence over the number of scans demanded. Due to the size of fixed costs, this is crucial for the determination of the cost of a scan, and hence for the economic evaluation. Flexibility has been considered both with respect to the timing of investment and the size of the project, the alternative being between acquiring only the PET scanner and buying the radiopharmaceutical from a different site, or investing in a Fully Equipped PET to produce the tracer on-site.

The cost-effectiveness evaluation has been extended to incorporate option values. With a dynamic programming approach, the minimum number of scans that ensures the cost-effectiveness of the examination provided under the two alternative modalities has been obtained as a function of the value of the incremental effectiveness. In our model, an examination provided with a scanner that uses the tracer produced on a different site can be cost-effective if the value of the incremental effectiveness is at least 418 euros. For this value, the scanner must be working at full capacity.

It has been assumed in the analysis that, if only a scanner is installed, the distance from the next FEP is such that the tracer can be bought from it. Moreover, the cost of the tracer has been estimated from a societal perspective. Under these assumptions, the results suggest that it is never optimal to provide PET scans with FEP if the number of examinations performed 
is below the scanner's maximum capacity. Above this level, the monetary value of incremental effectiveness must be at least 3,750 euros. More generally, from a regulator's standpoint the analysis seems to suggest that the installation of a new FEP should be very carefully evaluated, as long as more doses of the tracer can be produced in the FEPs already installed. If there is no regulatory power on the investment decision, hospitals face a further incentive to invest in FEP, because this enables them to sell the tracer.

This first attempt to extend the real option approach to the evaluation of health care technologies seems to suggest that this may well have an impact on investment decisions. Therefore, it is probably worth exploring the possibility of extending these techniques to other technologies. This could also lead to developments that enable researchers to take the peculiarities of evaluation in health care more carefully into account, for example with respect to the objectives of the different agents involved. Furthermore, this approach could be integrated with the insights coming from the literature on the value of information in cost-effectiveness analysis (Claxton, 1999), with the aim of relaxing the assumption of exogenous information and using this instrument to set priorities for research.

\section{Appendix}

Derivation of $F(n)$ : After the investment in FEP has been made, but before $\bar{n}_{f}$ is reached, the following Bellman equation applies:

$$
\rho\left(F(n)+I_{f}\right)=R_{f t}+\lim _{d t \rightarrow 0} \frac{1}{d t} E[d F(n)]
$$

Applying Ito's Lemma, the last term on the right hand side may be written as:

$$
\begin{aligned}
E[d F(n)]= & (1-\delta d t)\left[\mu n \frac{\partial F(n)}{\partial n}+\frac{1}{2} \sigma^{2} n^{2} \frac{\partial^{2} F(n)}{\partial^{2} n}\right]+ \\
& +\delta d t\left[-F(n)-I_{f}\right]+\circ(d t)
\end{aligned}
$$

The second term on the right hand side picks up the effect of $n$ dropping to 
zero. If this happens, the value of the project falls to $\left(-I_{f}\right)$. The substitution of this into eq. (22) yields the following differential equation:

$$
-(\rho+\delta)\left(F(n)+I_{f}\right)+\mu n \frac{\partial F(n)}{\partial n}+\frac{1}{2} \sigma^{2} n^{2} \frac{\partial^{2} F(n)}{\partial^{2} n}+R_{f t}=0
$$

The solution to this equation is typically made up of two parts (Dixit, 1994). The first is an exponential component associated with the option value. In general, this is equal to:

$$
D_{1} n^{\beta_{1}}+D_{2} n^{\beta_{2}}
$$

where, $\beta_{1}>1$ and $\beta_{2}<0$ are the solutions of the following equation:

$$
\frac{1}{2} \sigma^{2} \beta^{2}+\left(\mu-\frac{1}{2} \sigma^{2}\right) \beta-(\rho+\delta)=0
$$

The value of $D_{1}$ and $D_{2}$ is obtained imposing appropriate restrictions. First, for values of $n$ that tend to zero, the term $D_{2} n^{\beta_{2}}$ would make the value jump to infinity. Of course, this is inconsistent with our problem. Therefore, $D_{2}$ has to be set equal to zero. The second restriction follows from requiring continuity of the value function in $\bar{n}_{f}$. By setting $F\left(\bar{n}_{f}\right)=\bar{F}\left(\bar{n}_{f}\right)$, the value of $D_{1}$ is obtained (eq. 8).

The second part of the value function is the expected value of the project, ignoring the barriers. In this case, the relevant barrier is $\bar{n}_{f}$. If there was no chance that $n$ falls to zero, this expected value would be equal to (Huisman, 2001):

$$
\int_{0}^{\infty}\left(\lambda e-c_{f}\right) n_{t} e^{-\rho t} d t-\int_{0}^{\infty} f_{f} e^{-\rho t} d t=\frac{\left(\lambda e-c_{f}\right) n}{\rho-\mu}-\frac{f_{f}}{\rho}
$$

After the realization of the Poisson process at time $T$, net benefits fall to zero and, under our assumptions, yearly fixed costs need not be paid anymore. The expected value of this effect is,

$$
\begin{aligned}
& E\left[\frac{\left(\lambda e-c_{f}\right) n_{T}}{\rho-\mu} e^{-\rho T}-\frac{f_{f}}{\rho} e^{-\rho T}\right]= \\
& \frac{\left(\lambda e-c_{f}\right)}{\rho-\mu} E\left[n_{T} e^{-\rho T}\right]-\frac{f_{f}}{\rho} E\left[e^{-\rho T}\right]=
\end{aligned}
$$




$$
\begin{aligned}
& \frac{\left(\lambda e-c_{f}\right) n}{\rho-\mu} \int_{0}^{\infty} \delta e^{-\delta t} e^{-(\rho-\mu) t} d t-\frac{f_{f}}{\rho} \int_{0}^{\infty} \delta e^{-\delta t} e^{-\rho t}= \\
& \frac{\left(\lambda e-c_{f}\right) n}{\rho-\mu}\left(\frac{\delta}{\rho+\delta-\mu}\right)-\frac{f_{f}}{\rho}\left(\frac{\delta}{\rho+\delta}\right)
\end{aligned}
$$

Subtracting eq. (27) from eq. (26) we finally get the expected value component:

$$
\frac{\left(\lambda e-c_{f}\right) n}{\rho+\delta-\mu}-\frac{f_{f}}{\rho+\delta}
$$

Hence, the value function in this region is given by eq. (6).

The steps followed above show that only the positive root of eq. (7) appears in the value function. This is true also for the value functions that will be derived below. To simplify the notation, therefore, $\beta_{1}$ has been replaced with $\beta$ in the text.

Derivation of $\bar{P}(n)$ : For $n>\bar{n}_{p}$ the following Bellman equation applies:

$$
\rho\left(\bar{P}(n)+I_{p}\right)=\left(\lambda e-c_{p}\right) \bar{n}_{p}-f_{p}+\lim _{d t \rightarrow 0} \frac{1}{d t} E[d \bar{P}(n)]
$$

The same approach to the solution discussed for $F(n)$ may be followed. In this case, the second part of the value function is deterministic. The first part picks up the value of the option to expand the project to FEP. Hence, the value function is given by eq. (9).

Derivation of $P(n)$ : In principle, both types of barrier have to be taken into account in the determination of the value function after the investment in $\mathrm{P}$ has been made, as long as the limit to capacity has not been reached $(P(n))$. The endogenous barrier plays a role here because now, depending on the value of $n$, the option to expand the investment to FEP may be exercised. The interpretation of the exogenous barrier is instead the same as for $F(n)$, the only difference being that the maximum number of patients is now $\bar{n}_{p}<$ $\bar{n}_{f}$. Let us ignore, for the moment, the value of the opportunity to expand 
the investment. The corresponding partial value function may be obtained following exactly the same steps discussed for $F(n)$. This component of the value function will then be:

$$
C_{1} n^{\beta}+\frac{\left(\lambda e-c_{p}\right) n}{\rho+\delta-\mu}-\frac{f_{p}}{\rho+\delta}-\Phi_{p}-I_{p}
$$

$C_{1}$ is determined by imposing that, for $n=\bar{n}_{p}$, this expression equals the present value of the constant payoffs obtained for that value of the stochastic variable over the length of the project.

This function may now be extended to take the value of the opportunity to expand the investment into account. Consistent with the discussion above ${ }^{17}$, the full value function is given by the value obtained ignoring the endogenous barrier, plus the two non-linear terms associated with this:

$$
B_{1} n^{\beta_{1}}+B_{2} n^{\beta_{2}}+C_{1} n^{\beta}+\frac{\left(\lambda e-c_{p}\right) n}{\rho+\delta-\mu}-\frac{f_{p}}{\rho+\delta}-\Phi_{p}-I_{p}
$$

For the same reasons discussed above, $B_{2}$ has to be set equal to zero, and therefore, the value function in eq. (11) is obtained. The value of $B_{1}$ must be determined jointly with the value of the threshold $n_{f}^{*}$. As explained in Section 3.2 , this form of the value function would be actually relevant for $n \leq \bar{n}_{p}$ only if $n_{f}^{*}$ fell in this range.

Derivation of $\widetilde{P}(n)$ : It has been showed in the text that in our case the value matching and smooth pasting conditions yield no threshold such that $n_{f}^{*} \leq \bar{n}_{p}$. Therefore, the option to expand the project has no value for $n \leq \bar{n}_{p}$. The value function $\widetilde{P}(n)$ anticipates that there will be no investment before P's maximum capacity is reached. The expected value component is the same as in eq. (11). The exponential term picks up the impact on the value of the possibility that the upper bound $\bar{n}_{p}$ is reached. Hence, eq. (13) gives the value function for this case.

\footnotetext{
${ }^{17}$ See the derivation of $F(n)$.
} 


\section{References}

G. Barone-Adesi and R.E. Whaley. Efficient analytic approximation of American option values. Journal of Finance, 42:301-20, 1986.

M. Berger, M.K. Gould, and P.G. Barnett. The cost of Positron Emission Tomography in six United States Veterans Affairs hospitals and two academic medical centers. AJR, 181:359-65, 2003.

I. Bradbury, K. Facey, G. Laking, and P. Sharp. Investing in new technology: the PET experience. British Journal of Cancer, 89:224-27, 2003.

I. Brandbury, E. Bonell, J. Boynton, E. Cummins, K. Facey, K. Iqbal, G. Laking, C. McDonald, T. Parpia, P. Sharp, A. Single, and A. Walker. Positron Emission Tomography (PET) imaging in cancer management. Health Technology Board for Scotland. Health Technology Assessment Report 2, Glasgow, 2002.

CeVEAS. Indicazioni all'utilizzo della FDG-PET in oncologia. Dossier 81; Agenzia Sanitaria Regionale Emilia Romagna, 2003.

K. Claxton. The irrelevance of inference: a decision-making approach to the stochastic evaluation of health care technologies. Journal of Health Economics, 18:341-64, 1999.

M. Dietlein, K. Weber, A. Gandjour, D. Moka, P. Theissen, K.W. Lauterbach, and H. Schicha. Cost-effectiveness of FDG-PET for the management of solitary pulmonary nodules: a decision analysis based on cost reimbursement in Germany. European Journal of Nuclear Medicine, 27 (10):1441-56, 2000.

A. Dixit. The Art of Smooth Pasting. Harwood Academic, Chur, 1994.

A. Dixit and R.S. Pindyck. Investment Under Uncertainty. Princeton University Press, Princeton, 1994.

F.-P. Dussault, H. Van Nguyen, and F. Rachet. Positron Emission Tomography in Québec. Technical report, AÉTMIS, 2001. 
S. Eckermann and A.R. Willan. The option value of delay in Health Technology Assessment. The Centre for Applied Economic Research (CAER) working paper 6, 2006. http://www.caer.unsw.edu.au/CAER_WP_series.html, 2006.

S. Eckermann and A.R. Willan. Expected value of information and decision making in HTA. Health Economics, 16:195-209, 2007a.

S. Eckermann and A.R. Willan. Globally optimal trial design for local decision making. The Centre for Applied Economic Research (CAER) working paper 6, 2007. http://www.caer.unsw.edu.au/CAER_WP_series.html, 2007b.

Finger Lakes Health Systems Agency. Pet scanning in western New York. Niagara Health Quality Coalition, 2003.

S.S. Gambhir, J. Czernin, J. Schwimmer, D.H.S. Silverman, R.E. Coleman, and M.E. Phelps. A tabulated summary of the FDG PET literature. Journal of Nuclear Medicine, 42:1S-93S, 2001.

C.S. Hollenbeak, V.J. Lowe, and B.C.J. Stack. The cost-effectiveness of fluorodeoxyglucose 18-F Positron Emission Tomography in the NO Neck. Cancer, 92(9):2341-48, 2001.

K.J.M. Huisman. Technology Investment: a Game Theoretic Real Option Approach. Kluwer, Dodrecht, The Netherlands, 2001.

ICES. Health Technology Assessment of Positron Emission Tomography (PET) in Oncology- A Systematic Review. Institute for Clinical Evaluative Sciences, 2004.

P.B. Jacklin, S.F. Barrington, J.C. Roxburgh, G. Jackson, D. Sariklis, P.A. West, and M.N. Maisey. Cost-effectiveness of preoperative Positron Emission Tomography in ischemic heart disease. Ann Thorac Surg, 73:1403-10, 2002 . 
T. Klose, R. Leidl, I. Buchmann, H.J. Brambs, and S.N. Reske. Primary staging of lymphomas: cost-effectiveness of FDG-PET versus computed tomography. European Journal of Nuclear Medicine, 27(10):1457-64, 2000 .

O.K. Knudsen and P.L Scandizzo. An option approach to sustainable development. In World Bank working paper. World Bank, Washington D.C., 2002 .

P. Lasserre, J. Moatti, and A. Soubeyran. Early initiation of highly active antiretroviral therapies for AIDS: Dynamic choice with endogenous and exogenous learning. Journal of Health Economics, 25:579-98, 2006.

A. Müller, D. Stratmann-Schöne, T. Klose, and R. Leidl. Overview of economic evaluation of Positron-Emission Tomography. European Journal of Economics, 3(1):59-65, 2002.

S. Palmer and P.C. Smith. Incorporating option values into the economic evaluation of health care technologies. Journal of Health Economics, 19 (5):755-66, 2000.

K.LK. Reiter, D.J. Smith, J.R.C. Wheeler, and H. Rivenson. Capital investment strategies in health care systems. Journal of Health Care Finance, 26(4):31-41, 2000 .

G. Robert and R. Milne. Positron Emission Tomography: establishing priorities for health technology assessment. Health Technology Assessment, 3(16), 1999.

E.S. Schwartz and L. Trigeorgis. Real options and investment under uncertainty: classical readings and recent contributions. MIT press, Cambridge (MA), 2001.

S. Titman. Urban land prices under uncertainty. The American Economic Review, 75(3):505-14, 1985. 